УДК 548.1

ISSN 1606-867X

DOI: https://doi.org/10.17308/kcmf.2019.21/2361

Поступила в редакцию 14.11.2019

Подписана в печать 15.12.2019

\title{
Строение специальных межкристаллитных границ в двухкомпонентных кристаллах
}

\author{
(c) 2019 Б. М. Даринский ${ }^{\varpi}$, Н. Д. Ефанова, А. С. Прижимов \\ Воронежский государственный университет \\ Университетская пл., 1, 394018 Воронеж, Российская Федерация
}

\begin{abstract}
Аннотация. В настоящей работе представлена новая методика построения решетки совпадающих узлов для кристаллов простой кубической, ОЦК, ГЦК структур, имеющих моноэлементные и полиэлементные составы. Разработан метод нахождения атомов различных элементов в межкристаллитных границах на основе специально построенной кристаллографической группы. Указаны возможные элементные составы специальных межкристаллитных границ, зарядовые состояния сопрягающихся плоскостей.
\end{abstract}

Ключевые слова: решетка совпадающих узлов, межфазные границы, кристалл, специальные границы, структура.

\section{ВВЕДЕНИЕ}

Специальные границы между кристаллитами выделяются из всей совокупности межкристаллитных границ, прежде всего из-за их повышенной устойчивости, что связано с их пониженной энергией. Интерес к этим границам продолжается от первых работ шестидесятых годов прошлого века [1-5] по настоящее время [6-16]. Важным направлением, связанным со знаниями в области структуры и свойств межкристаллитных границ и возникшим в последние два десятилетия, является целенаправленное приготовление поликристаллов, имеющих заданную геометрию размещения отдельных кристаллитов и межкристаллитных границ (Grain Boundary Engineering) как в трехмерных $[17,18]$, так и в пленочных образцах [19]. Надежды на успех в решении этих задач основаны на существенном развитии методов направленной кристаллизации, в частности, в наноразмерных материалах [20]. Исследования строения и свойств межкристаллитных границ в кристаллах, содержащих два или более элементов, ионных кристаллах проводятся с целью понимания влияния их строения на доменную структуру, электронную и ионную электрическую проводимость [20], характеристики барьера Шоттки [21], распределения примесных атомов в границе и вблизи нее

Даринский Борис Михайлович, e-mail: darinskii@mail.ru
[22]. Все эти эффекты существенным образом зависят от электрического поля, порождаемого ионной структурой межкристаллитной границы [23-25]. Поэтому следует полагать, что исследование строения границ и их совокупности в поликристаллических образцах является актуальным. В предыдущих работах авторов [26] была развита методика, позволяющая классифицировать совокупность специальных границ в кристаллах, имеющих простую кубическую ОЦК и ГЦК решетки. В настоящей работе аналогичная задача решается для кристаллов со структурой $\mathrm{NaCl}$ и CsCl, указываются геометрические условия формирования решеток совпадения и совокупность параметров разориентации кристаллитов, имеющих общую решетку совпадающих узлов и разные структуры межкристаллитных границ, различающиеся элементным составом и зарядовым состояниями.

\section{МЕТОДИКА РАСЧЕТОВ}

В качестве исходных элементов структуры кристаллической решетки выберем решеточные векторы $\mathbf{B}_{0}$ и $\mathbf{B}$, принадлежащие общему координационному многограннику, построенному вокруг узла в вершине ячейки кубической решетки и поэтому имеющие одинаковые длины. Операция поворота твердого тела определяется вращением вектора $\mathbf{B}_{0}$ до совпадения с вектором $\mathbf{B}_{0}$ вокруг оси $\mathbf{A}[26]$. Этот вектор лежит в

Контент доступен под лицензией Creative Commons Attribution 4.0 License.

The content is available under Creative Commons Attribution 4.0 License. 
кристаллографической плоскости, перпендикулярной вектору $\mathbf{B}-\mathbf{B}_{0}$. Такая плоскость определяется базисными векторами:

$$
\mathbf{A}_{n}=\left[\mathbf{B}_{\mathbf{0}} \mathbf{B}\right], \mathbf{A}_{c}=\mathbf{B}+\mathbf{B}_{\mathbf{0}}
$$

Отметим, что $\mathbf{A}_{n}$ совпадает по направлению с вектором обратной решетки, построенном на векторах $\mathbf{B}$ и $\mathbf{B}_{0} \mathbf{A}_{c}$, лежит на биссектрисе этих векторов. После проведения операций (1), возможно, следует сократить результат, так, чтобы векторы $\mathbf{A}_{n}$ и $\mathbf{A}_{c}$ стали минимальными решеточными векторами. В качестве примера рассмотрим поворот, который совмещает векторы [100] и [010].

Совокупность всех осей вращения определяется выражением:

$$
\varphi=a_{n} \mathbf{A}_{n}+a_{c} \mathbf{A}_{c}
$$

где $a_{n}$ и $a_{c}$ - целые числа, выбор которых позволяет однозначно определить вектор А.

Угол поворота $\varphi$ определяется следующей формулой для $\cos \varphi[26]$ :

$$
\begin{gathered}
\cos (\varphi)=\frac{\left(\mathbf{B}_{0} \mathbf{B}\right) A^{2}-(\mathbf{A B})^{2}}{B^{2} A^{2}-(\mathbf{A B})^{2}}, \\
\mathbf{B}=n_{1} \mathbf{a}_{1}+n_{2} \mathbf{a}_{2}+n_{3} \mathbf{a}_{3}, \\
\mathbf{B}_{0}=m_{1} \mathbf{a}_{1}+m_{2} \mathbf{a}_{2}+m_{3} \mathbf{a}_{3},
\end{gathered}
$$

где $n_{i}, m_{i}$ - целые числа для примитивной кубической решетки. Совокупность $m_{i}$ получается перестановкой чисел $n_{1}, n_{2}, n_{3}$ и изменением их знака. В нашем случае векторы $\mathbf{a}_{i}$ будут совпадать по направлениям с ортами $\mathbf{i}, \mathbf{j}, \mathbf{k}$ декартовой системы координат и выражаться через них следующим образом:

$$
a_{1}=a \mathbf{i}, \mathbf{a}_{2}=a \mathbf{j}, \mathbf{a}_{3}=a \mathbf{k}
$$

где $a$ - длина ребра кубической ячейки, в дальнейшем она будет считаться равной 1.

В качестве третьего вектора решетки совпадений выберем вектор:

$$
\mathbf{C}=[\mathrm{AB}],
$$

Поскольку векторы А и В имеют целочисленные координаты, вектор $\mathbf{C}$ тоже будет иметь целочисленные координаты, поэтому его можно принять в качестве третьего вектора решетки совпадений. В узел, определяемый вектором $\mathbf{C}$, попадает вектор:

$$
\mathbf{C}_{0}=\left[\mathrm{AB}_{0}\right] .
$$

Если в результате вычислений численных значений этих векторов окажется, что они имеют общий целочисленный сомножитель, то на него нужно будет сократить так, чтобы эти векторы имели минимальную длину.

Совокупности векторов $\mathbf{A}, \mathbf{B}_{0}, \mathbf{C}_{0}$ и $\mathbf{A}, \mathbf{B}, \mathbf{C}$, построенных таким образом, определяют две элементарные ячейки, которые после поворота вокруг оси А совпадут друг с другом и породят решетку совпадения (рис. 1).

Рассмотрим совокупность точек в ячейке кристаллической решетки, имеющих нулевые и полуцелые индексы:

$$
\begin{gathered}
\mathrm{I}=[000], \\
\mathrm{E}_{1}=\left[\frac{1}{2} 00\right], \mathrm{E}_{2}=\left[0 \frac{1}{2} 0\right], \mathrm{E}_{3}=\left[00 \frac{1}{2}\right](\text { Edge }) ; \\
\mathrm{F}_{1}=\left[0 \frac{1}{2} \frac{1}{2}\right], \mathrm{F}_{2}=\left[\frac{1}{2} 0 \frac{1}{2}\right], \mathrm{F}_{3}=\left[\frac{1}{2} \frac{1}{2} 0\right] \text { (Face);} \\
\mathrm{C}=\left[\frac{1}{2} \frac{1}{2} \frac{1}{2}\right](\text { Centre }) .
\end{gathered}
$$

Эта совокупность естественным образом разбивается на четыре типа. Точка I располагается в вершине ячейки, точка C - в ее центре, $\mathrm{E}_{1}$, $\mathrm{E}_{2}, \mathrm{E}_{3}$ - в серединах ребер куба, $\mathrm{F}_{1}, \mathrm{~F}_{2}, \mathrm{~F}_{3}$ - в центрах граней.

В кристалле каждому типу точек можно сопоставить определенный химический элемент. В частности в тривиальном случае простой кубической решетки всем точкам кроме вершины куба сопоставляются вакансии. В ОЦК кристаллах точкам типа I и могут соответствовать либо одинаковые атомы, либо разные элементы таблицы Менделеева. В последнем случае получится структура CsCl. Остальным точкам соответствуют вакансии. В ГЦК кристаллах возможно расположение двух разных элементов по точкам I и F. В кристаллах, содержащих в своем составе три или четыре элемента, заполнение точек составляющими элементами однозначно определяется их структурой.

Для нахождения возможных структур специальных межкристаллитных границ введем груп-

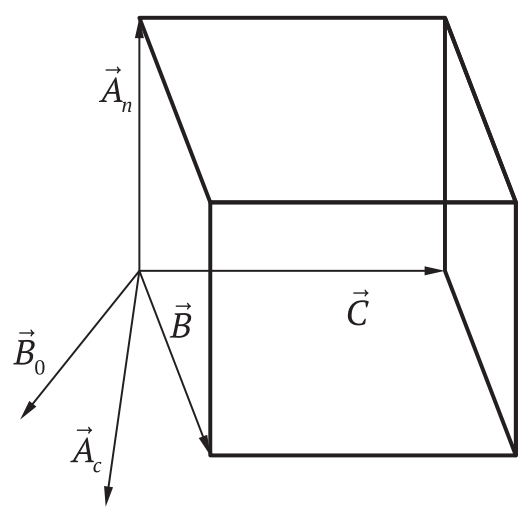

Рис. 1. Элементарная ячейка решетки совпадающих узлов

[Fig. 1. Elementary cell of the lattice of matching nodes] 
пу D (displacement) смещений решетки, состоящую из операций, приведенных выше. Каждая из них представляет собой добавление соответствующих компонент к компонентам исходной точки с последующим обнулением целочисленных компонент. Правила группового умножения представлены в таблице.

Введенная группа D является одной из подгрупп пространственной группы кристаллов и имеет конечный порядок. В этой группе все операции коммутативны, каждый элемент группы имеет цикл, равный двум, элементы $\mathrm{E}_{1}, \mathrm{E}_{2}, \mathrm{E}_{3}$ образуют генератор группы, остальные элементы группы получаются перемножением: $\mathrm{F}_{1}=\mathrm{E}_{2} \mathrm{E}_{3}$, $\mathrm{F}_{2}=\mathrm{E}_{1} \mathrm{E}_{3}, \mathrm{~F}_{3}=\mathrm{E}_{1} \mathrm{E}_{2}, \mathrm{C}=\mathrm{E}_{1} \mathrm{E}_{2} \mathrm{E}_{3}$. Группа $\mathrm{D}$ содержит семь подгрупп второго и семь подгрупп четвертого порядка. Семь подгрупп второго порядка распадаются на три типа: $\left\{\mathrm{I}, \mathrm{E}_{1}\right\},\left\{\mathrm{I}, \mathrm{F}_{1}\right\},\{\mathrm{I}, \mathrm{C}\}$. Подгруппы первых двух типов получаются сменой индекса от единицы до трех. Семь подгрупп четвертого порядка группы D также можно разделить на три типа $\left\{\mathrm{I}, \mathrm{E}_{1}, \mathrm{E}_{2}, \mathrm{~F}_{3}\right\},\left\{\mathrm{I}, \mathrm{E}_{1}, \mathrm{~F}_{1}, \mathrm{C}\right\},\left\{\mathrm{I}, \mathrm{F}_{1}\right.$, $\left.\mathrm{F}_{2}, \mathrm{~F}_{3}\right\}$. Три подгруппы первого типа получаются перестановкой индексов, а второго типа их сменой от единицы до трех. Отметим, что каждый тип подгрупп можно рассматривать как базис группы перестановок, при этом первые два типа образуют представление третьего порядка, а последний является инвариантом.

Рассмотрим строение кристаллографических плоскостей в аспекте их групповых свойств и элементного состава. Зададим кристаллографическую плоскость двумя определяющими векторами с целочисленными компонентами $\left(\mathrm{n}_{1}, \mathrm{n}_{2}\right.$, $\left.\mathrm{n}_{3}\right),\left(\mathrm{m}_{1}, \mathrm{~m}_{2}, \mathrm{~m}_{3}\right)$, исходящими из начала координат. Эти тройки чисел не имеют общего множителя. Для них имеются три варианта возможных значений: одно нечетное и два четные, два нечетных одно четное и все три числа нечетные.
В средней точке этих векторов будет располагаться одна из точек с полуцелыми компонентами. В первом варианте будет точка типа $\mathrm{E}_{1}$, во втором $\mathrm{F}_{1}$, в третьем С. В середине ячейки этой плоскости будет находиться точка, тип которой определяется как произведение элементов группы D, соответствующих серединам ребер. B peзультате получается, что плоская группа симметрии любой кристаллографической плоскости является одной из указанных выше подгрупп четвертого порядка группы D. Каждому смещению группы D можно поставить в соответствие химический элемент, совокупность которых определяется брутто формулой кристалла. В каждой из кристаллографических плоскостей будут находиться узлы разных элементов, совокупность которых будет определять суммарный электрический заряд плоскости. Для нахождения знака заряда можно воспользоваться представлениями о степени окисления ионов в элементарной ячейке плоскости.

Рассмотрим структуру плоскостей в разных кубических решетках. Для простой кубической решетки имеется только единичный элемент группы D. Все параллельные плоскости имеют одинаковую структуру.

Для ОЦК решетки имеется подгруппа, состоящая из двух элементов $\{\mathrm{I}, \mathrm{C}\}$ группы D. Результаты смещения плоскостей на половину пространственной диагонали представлены ниже:

$$
\begin{gathered}
\left\{\mathrm{I}, \mathrm{E}_{1}, \mathrm{E}_{2}, \mathrm{~F}_{3}\right\} \rightarrow\left\{\mathrm{C}, \mathrm{F}_{1}, \mathrm{~F}_{2}, \mathrm{E}_{3}\right\}, \\
\left\{\mathrm{I}, \mathrm{F}_{1}, \mathrm{~F}_{2}, \mathrm{~F}_{3}\right\} \rightarrow\left\{\mathrm{C}, \mathrm{E}_{1}, \mathrm{E}_{2}, \mathrm{E}_{3}\right\}, \\
\left\{\mathrm{I}, \mathrm{E}_{1}, \mathrm{~F}_{1}, \mathrm{C}\right\} \rightarrow\left\{\mathrm{I}, \mathrm{E}_{1}, \mathrm{~F}_{1}, \mathrm{C}\right\} .
\end{gathered}
$$

Они показывают, структура плоскостей, проходящих через узлы и центры куба, не изменяется. Узлы решетки, через которые проходят плоскости первых двух типов меняют свое окружение. Применим эти результаты к решетке хло-

Таблица 1. Правила перемножения элементов группы D смещений решетки

[Table 1. Rules of multiplication of the elements of D group of lattice displacements]

\begin{tabular}{|c|c|c|c|c|c|c|c|c|}
\hline $\mathrm{D}$ & $\mathrm{I}$ & $\mathrm{E}_{1}$ & $\mathrm{E}_{2}$ & $\mathrm{E}_{3}$ & $\mathrm{~F}_{1}$ & $\mathrm{~F}_{2}$ & $\mathrm{~F}_{3}$ & $\mathrm{C}$ \\
\hline $\mathrm{I}$ & $\mathrm{I}$ & $\mathrm{E}_{1}$ & $\mathrm{E}_{2}$ & $\mathrm{E}_{3}$ & $\mathrm{~F}_{1}$ & $\mathrm{~F}_{2}$ & $\mathrm{~F}_{3}$ & $\mathrm{C}$ \\
\hline $\mathrm{E}_{1}$ & $\mathrm{E}_{1}$ & $\mathrm{I}$ & $\mathrm{F}_{3}$ & $\mathrm{~F}_{2}$ & $\mathrm{C}$ & $\mathrm{E}_{3}$ & $\mathrm{E}_{2}$ & $\mathrm{~F}_{1}$ \\
\hline $\mathrm{E}_{2}$ & $\mathrm{E}_{2}$ & $\mathrm{~F}_{3}$ & $\mathrm{I}$ & $\mathrm{F}_{1}$ & $\mathrm{E}_{3}$ & $\mathrm{C}$ & $\mathrm{E}_{1}$ & $\mathrm{~F}_{2}$ \\
\hline $\mathrm{E}_{3}$ & $\mathrm{E}_{3}$ & $\mathrm{~F}_{2}$ & $\mathrm{~F}_{1}$ & $\mathrm{I}$ & $\mathrm{E}_{2}$ & $\mathrm{E}_{1}$ & $\mathrm{C}$ & $\mathrm{F}_{3}$ \\
\hline $\mathrm{F}_{1}$ & $\mathrm{~F}_{1}$ & $\mathrm{C}$ & $\mathrm{E}_{3}$ & $\mathrm{E}_{2}$ & $\mathrm{I}$ & $\mathrm{F}_{3}$ & $\mathrm{~F}_{2}$ & $\mathrm{E}_{1}$ \\
\hline $\mathrm{F}_{2}$ & $\mathrm{~F}_{2}$ & $\mathrm{E}_{3}$ & $\mathrm{C}$ & $\mathrm{E}_{1}$ & $\mathrm{~F}_{3}$ & $\mathrm{I}$ & $\mathrm{F}_{1}$ & $\mathrm{E}_{2}$ \\
\hline $\mathrm{F}_{3}$ & $\mathrm{~F}_{3}$ & $\mathrm{E}_{2}$ & $\mathrm{E}_{1}$ & $\mathrm{C}$ & $\mathrm{F}_{2}$ & $\mathrm{~F}_{1}$ & $\mathrm{I}$ & $\mathrm{E}_{3}$ \\
\hline $\mathrm{C}$ & $\mathrm{C}$ & $\mathrm{F}_{1}$ & $\mathrm{~F}_{2}$ & $\mathrm{~F}_{3}$ & $\mathrm{E}_{1}$ & $\mathrm{E}_{2}$ & $\mathrm{E}_{3}$ & $\mathrm{I}$ \\
\hline
\end{tabular}


рида цезия. Узлам I поставим в соответствие ион хлора, в узлах С поместим ион цезия. Результаты показывают, что смещение плоскостей первого и второго типов сопровождается сменой их элементного состава. При этом исходная плоскость, проходящая через ионы хлора, сменяется аналогичной плоскостью, проходящей через ионы цезия. Плоскости последнего типа после этой операции химический состав не меняют. Таким образом, первые два типа плоскостей содержат заряды разных знаков, а последний тип плоскостей соответствует незаряженным плоскостям.

Решетка каменной соли. Будем полагать, что в узлах $\mathrm{I}, \mathrm{F}_{1}, \mathrm{~F}_{2}, \mathrm{~F}_{3}$ располагаются ионы хлора, в узлах $C, \mathrm{E}_{1}, \mathrm{E}_{2}, \mathrm{E}_{3}$ - ионы натрия. Смещение плоскостей типа $\left\{\mathrm{I}, \mathrm{F}_{1}, \mathrm{~F}_{2}, \mathrm{~F}_{3}\right\}$, имеющих химический состав $\mathrm{NaCl}_{3}$, приводит к появлению плоскости $\mathrm{Na}_{3} \mathrm{Cl}$. Обе плоскости имеют ненулевые степени окисления, являются носителями электрического заряда, поэтому система параллельных плоскостей такого типа сопровождается появлением электрических полей, имеющих отличную от нуля нормальную компоненту напряженности электрического поля. Системы плоскостей $\left\{\mathrm{I}, \mathrm{E}_{1}\right.$, $\left.\mathrm{E}_{2}, \mathrm{~F}_{3}\right\},\left\{\mathrm{I}, \mathrm{E}_{1}, \mathrm{~F}_{1}, \mathrm{C}\right\}$ оказываются незаряженными, поэтому соответствующие межкристаллитные границы являются устойчивыми.

\section{РЕЗУЛЬТАТЫ И ИХ ОБСУЖДЕНИЕ}

Далее изложим методику определения элементного состава межкристаллитных границ на примере межкристаллитных границ компланарных граням ячейки решетки совпадений, имеющих наиболее плотную упаковку.

Элементный состав граней ячейки решетки совпадающих узлов определим путем попарного выбора векторов A, B и $\mathbf{C}$ и последующего нахождения узлов разных типов, лежащих в плоскости выбранной грани ячейки. Будем помечать каждую грань ячейки решетки совпадений вектором ячейки, не принадлежащим этой грани. Все точки грани $\vec{C}$ ячейки решетки совпадений могут быть определены линейной комбинацией $\mathrm{aA}+\mathrm{bB}$, в которой коэффициенты а и $\mathrm{b}$ принадлежат сегменту [0,1). Точки, являющиеся узлами кристаллографической плоскости кристалла, определяются подходящим выбором рациональных коэффициентов a и b. Элементный состав грани определяется типом узлов, лежащих в этой грани. Аналогичным способом находится элементный состав других граней.

Параллельный перенос кристаллографической плоскости решетки совпадающих узлов приводит к изменению ее элементного состава. Для нахождения закономерностей этого изменения введем группу D. Межкристаллитные границы могут быть получены путем контактирования каждой из плоскостей двух контактирующих полукристаллов. Среди всего набора пар контактирующих плоскостей найдется пара с наименьшей энергией. Остальные контакты следует рассматривать как метастабильные межкристаллитные границы.

В качестве примера рассмотрим поворот, при котором вектор при котором вектор В $_{0}$ с компонентами [100] переходит в вектор В с компонентами [010]. Для этой пары векторов получаем $\mathbf{A}_{n}=$ [001] и $\mathbf{A}_{c}=$ [110]. В качестве оси поворота выберем нетривиальную ось минимальной длины $\mathbf{A}=$ [112]. Векторы $\mathbf{C}_{0}$ и $\mathbf{C}$ находим, используя (6) и (7), получим $\mathbf{C}_{0}=[02 \overline{1}], \mathbf{C}=[\overline{2} 01]$.

Рассмотрим грань $\mathbf{C}_{0}$. Из линейной комбинации $\mathrm{aA}+\mathrm{b}_{0} \mathbf{B}_{0}=\mathrm{a}[112]+\mathrm{b}_{0}[100]$ видно, что при соответствующих коэффициентах $\left(\mathrm{a}, \mathrm{b}_{0}\right)$ эта кристаллографическая плоскость проходит через ионы хлора и заряжена отрицательно. Аналогично, плоскость С проходит через ионы хлора. Соседняя плоскость, проходящая через ионы цезия, имеет такую же кристаллографическую структуру и положительный электрический заряд. Таким образом, система плоскостей С представляет собой чередующиеся заряженные плоскости. Аналогичные вычисления для плоскости А показали, что они относится к типу $\left\{\mathrm{I}_{1} \mathrm{E}_{1}, \mathrm{E}_{2}\right.$, $\left.\mathrm{F}_{3}\right\}$, поэтому эти системы плоскостей также будут иметь электрическое поле. А плоскости В и $\mathbf{B}_{0}$ относятся к типу $\left\{\mathrm{I}, \mathrm{E}_{1}, \mathrm{~F}_{1}, \mathrm{C}\right\}$ и электрически нейтральны.

\section{ЗАКЛЮЧЕНИЕ}

Разработанный метод построения решеток совпадающих узлов в одноэлементных кристаллах кубической структуры распространен на кристаллы, имеющие в своем составе более одного химического элемента. Разработан метод нахождения расположений атомов разных элементов в специальных межкристаллитных границах на основе специально сконструированной кристаллографической группы смещений. Дана классификация возможных вариантов распределения элементов по плоскостям и зарядам плоскостей в зависимости от их ориентации.

\section{КОНФЛИКТ ИНТЕРЕСОВ}

Авторы декларируют отсутствие явных и потенциальных конфликтов интересов, связанных с публикацией настоящей статьи. 


\section{СПИСОК ЛИТЕРАТУРЫ}

1. Bollmann W. On the geometry of grain and phase boundaries // Phil. Mag., 1967, v. 16(140), pp. 363-381. DOI: https://doi.org/10.1080/14786436708229748

2. Bollmann W. On the geometry of grain and phase boundaries // Phil. Mag., 1967, v. 16(140), pp. 383-399. https://doi.org/10.1080/14786436708229749

3. Grimmer H. A method of determining the coincidence site lattices for cubic crystals // Acta Cryst. A, 1974, v. 30(2), pp. 680-680. DOI: https://doi. org/10.1107/s056773947400163x

4. Grimmer H., Bollmann W., Warrington D. T. Coincidence-site lattices and complete pattern-shift in cubic crystals // Acta Cryst. A, 1974, v. 30(2), pp. 197207. DOI: https://doi.org/10.1107/ s056773947400043x

5. Орлов А. Н., Перевезенцев В. Н., Рыбин В. В. Границы зерен в металлах. М.: Металлургия, 1980, $224 \mathrm{c}$.

6. Глейтер Г., Чалмерс Б. Большеугловые границы зерен. М.: Мир, 1975, 376 с.

7. Страумал Б. Б., Швиндлерман Л. С. Термическая стабильность и области существования специальных границ зерен // Поверхность. Физика, химия, механика, 1986, т. 10, с. 5-14.

8. Fortes M. A. Coincidence site lattices in noncubic lattices // Phys. Stat. Sol. B, 1977, v. 82(1). pp. 377-382. DOI: https://doi.org/10.1002/ pssb.2220820143

9. Bonnet R., Durand F. A general analytical method to find a basis for the DSC lattice // Scripta Met., 1975, v. 9(9), pp. 935-939. DOI: https://doi. org/10.1016/0036-9748(75)90548-7

10 . Bonnet R. Note on a general analytical method to find a basis for the DSC lattice. Derivation of a basis for the CSL// Scripta Met., 1976, v. 10(9), pp. 801-806. DOI: https://doi.org/10.1016/0036-9748(76)90297-0

11. Bonnet R., Cousineau E. Computation of coincident and near-coincident cells for any two lattices - related DSC-1 and DSC-2 lattices // Acta Cryst. A, 1977, v. 33(5), pp. 850-856. DOI: https://doi. org/10.1107/s0567739477002058

12. Рыбин В. В., Перевезенцев В. Н. // ФТТ, 1975, т. 17 , с. $3188-3193$.

13. Андреева А. В., Фионова Л. К. Анализ межкристаллитных границ на основе теории решеток совпадающих узлов // ФММ, 1977, т. 44, с. 395400.

14. Кайбышев О. А., Валиев Р. З. Границы зерен и свойства металлов. М.: Металлургия, 1987, 214 c.
15. Копецкий Ч. В., Орлов А. Н., Фионова Л. К. Границы зерен в чистых материалах. М.: Наука, 1987, $160 \mathrm{c.}$

16. Бокштейн Б. С. Структура и свойства внутренних поверхностей раздела в металлах. М.: Металлургия, 1988, 272 с.

17. Kobayashi S., Tsurekawa S., Watanabe T. A new approach to grain boundary engineering for nanocrystalline materials // Beilstein J. Nanotechnol., 2016, v. 7, pp. 1829-1849. DOI: https://doi.org/10.3762/bjnano.7.176

18. Сухомлин Г. Д. Специальные границы в феррите низкоуглеродистых сталей // Металлофизика, новейшие технологии, 2013, т. 35, с. 12371249.

19. Watanabe T. Grain boundary engineering: historical perspective and future prospects // Journal of Materials Science, 2011, v. 46, pp. 4095-4115. DOI: https://doi.org/10.1007/s10853-011-5393-z

20. Waser R. Electronic properties of grain boundaries in $\mathrm{SrTiO}_{3}$ and $\mathrm{BaTiO}_{3}$ ceramics // Solid State Ionics, 1995, v. 75, pp. 89-99. DOI: https://doi. org/10.1016/0167-2738(94)00152-i

21. Daniels J., Wemicke R. New Aspects of an Improved PTC Model // Philips Res. Rep., 1976, v. 31, pp. 544-559.

22. Vikrant K. S. N., Edwin G. R. Charged grain boundary transitions in ionic ceramics for energy applications // Computational Materials, 2019, v. 5(1), pp. 24. DOI: https://doi.org/10.1038/s41524-019$0159-2$

23. Kim M., Duscher G., Browning N.D., Sohlberg K., Pantelides S. T., Pennycook S. J. Nonstoichiometry and the electrical activity of grain boundaries in $\mathrm{SrTiO}_{3} / /$ Physical Review Letters, 2001, v. 86, pp. 4056-4059. DOI: https://doi.org/10.1103/physrevlett.86.4056

24. Oyama T., Wada N., Takagi H. Trapping of oxygen vacancy at grain boundary and its correlation with local atomic configuration and resultant excess energy in barium titanate: A systematic computational analysis // Physical Review B, 2010, v. 82, pp. 134107. DOI: https://doi.org/10.1103/physrevb.82.134107

25. Duffy D.M., Tasker P.W. Space-charge regions around dipolar grain boundaries // Journal of Applied Physics, 1984, v. 56, pp. 971-977. DOI: https://doi. org/10.1063/1.334037

26. Даринский Б. М., Ефанова Н. Д., Прижимов А. С. Систематика решеток совпадающих узлов для ОЦК и ГЦК кристаллов // Конденсированные среды и межфазные границы, 2018, т. 20(4), с. 581586. DOI: https://doi.org/10.17308/kcmf.2018.20/632 


\title{
Structure of the Special Intercrystalline Boundaries in Two Component Crystals
}

\author{
(C) 2019 B. M. Darinsky ${ }^{\bowtie}$, N. D. Efanova, A. S. Prizhimov \\ Vorornezh State University \\ 1, Universitetskaya pl., 394018 Voronezh, Russian Federation
}

\begin{abstract}
Object. The object of the study was the intercrystalline boundaries with a periodic atomic structure in two-component cubic crystals. Special boundaries are characterized by increased thermodynamic stability due to the relatively low energy of formation and specific electrical characteristics, such as Schottky barriers. Therefore, they are of great interest to researchers and developers of materials and devices. This study was carried out in the grain boundary engineering direction based on ion crystals. Aim of the study. The goal of the study was the atomic structure of these boundaries, classification of intercrystalline boundaries based on their elemental composition, and the evaluation of intercrystalline boundaries as sources of electric fields in the crystal volume.

Methods and methodology. As a method of research, the ideas of crystallographic symmetry of lattices having a simple, face-centred, and bulk-centred geometric structure were used.

Results. A new method was developed for the appliance of lattice sites to certain elements of chemical composition using a specially constructed crystallographic group called the group of displacements. Specific groups of displacements for crystals of BCC, FCC, and simple cubic structure with two-component chemical composition were constructed. Based on this, the conditions determining the families of planes with the same elemental compositions and the relative arrangement of elements in the intercrystalline contact were formulated. Families of neutral atomic planes and families containing excess positive and negative charges were specified.

Conclusion. The technique of determining the sequence of alternation of these planes in the intercrystalline boundary region was described. $\mathrm{NaCl}, \mathrm{CsCl}$, and other crystals are considered as examples. For each crystal family, the orientations of the charged and neutral planes were indicated.
\end{abstract}

Keywords: coincidence site lattice, interfaces, crystal, special boundaries, structure.

\section{CONFLICT OF INTEREST}

The authors declare the absence of obvious and potential conflicts of interest related to the publication of this article.

\section{REFERENCES}

1. Bollmann W. On the geometry of grain and phase boundaries. Phil. Mag., 1967, v. 16(140), pp. 363-381. DOI: https://doi.org/10.1080/14786436708229748

2. Bollmann W. On the geometry of grain and phase boundaries. Phil. Mag., 1967, v. 16(140), pp. 383-399. https://doi.org/10.1080/14786436708229749

3. Grimmer H. A method of determining the coincidence site lattices for cubic crystals. Acta Cryst. A, 1974, v. 30(2), pp. 680-680. DOI: https://doi. org/10.1107/s056773947400163x

4. Grimmer H., Bollmann W., Warrington D. T. Coincidence-site lattices and complete pattern-shift in cubic crystals. Acta Cryst. A, 1974, v. 30(2), pp. 197-

Boris M. Darinskiy, e-mail: darinskii@mail.ru
207. DOI: https://doi.org/10.1107/ s056773947400043x

5. Orlov A. N., Perevezentsev V. N., Rybin V. V. Grain boundaries in metals. Moscow, Metallurgy Publ., 1980, 224 p. (in Russ.)

6. Gleyter G., Chalmers B. High-angle Ggrain Bdoundaries. Moscow, Mir Publ., 1975, 376 p. (in Russ.)

7. Straumal B. B., Shvindlerman H. P. Thermal stability and regions of existence of special grain boundaries. Surface. Physics, Chemistry, Mechanics, 1986, v. 10, pp. 5-14. (in Russ.)

8. Fortes M. A. Coincidence site lattices in noncubic lattices. Phys. Stat. Sol. B, 1977, v. 82(1). pp. 377-382. DOI: https://doi.org/10.1002/ pssb.2220820143

9. Bonnet R., Durand F. A general analytical method to find a basis for the DSC lattice. Scripta Met., 1975, v. 9(9), pp. 935-939. DOI: https://doi. org/10.1016/0036-9748(75)90548-7 
10. Bonnet R. Note on a general analytical method to find a basis for the DSC lattice. Derivation of a basis for the CSL. Scripta Met., 1976, v. 10(9), pp. 801-806. DOI: https://doi.org/10.1016/0036-9748(76)90297-0

11. Bonnet R., Cousineau E. Computation of coincident and near-coincident cells for any two lattices - related DSC-1 and DSC-2 lattices. Acta Cryst. A, 1977, v. 33(5), pp. 850-856. DOI: https://doi. org/10.1107/s0567739477002058

12. Rybin V. V., Perevezentsev V. N. FTT [Technical Physics], 1975, v. 17, pp. 3188-3193. (in Russ.)

13. Andreeva A. V., Fionova L. K. Analysis of intergranular boundaries based on the theory of lattices of matching nodes. Fizika Metallov $i$ Metallovedenie [Physics of Metals and Metallography], 1977, v. 44, pp. 395-400. (in Russ.)

14. Kaibyshev O. A., Valiev R. Z. Grain boundaries and properties of metals. Moscow, Metallurgy Publ., 1987, 214 p. (in Russ.)

15. Kopetsky H. V., Orlov A. N., Fionova L. K. Grain boundaries in pure materials. Moscow, Nauka Publ., 1987,160 p. (in Russ.)

16. Bokshtein B. S. Structure and properties of internal interfaces in metals. Moscow, Metallurgy Publ., 1988, 272 p. (in Russ.)

17. Kobayashi S., Tsurekawa S., Watanabe T. A new approach to grain boundary engineering for nanocrystalline materials. Beilstein J Nanotechnol., 2016(7), pp. 1829-1849. DOI: https://doi.org/10.3762/bjnano.7.176

18. Sukhomlin G. D. Special boundaries in low carbon steel ferrite. Metallofiz. Noveishie Tekhnol.2013, v. 35, pp. 1237-1249. (in Russ.)

19. Watanabe T. Grain boundary engineering: historical perspective and future prospects. Journal of
Materials Science, 2011, v. 46, pp. 4095-4115. DOI: https://doi.org/10.1007/s10853-011-5393-z

20. Waser R. Electronic properties of grain boundaries in $\mathrm{SrTiO}_{3}$ and $\mathrm{BaTiO}_{3}$ ceramics. Solid State Ionics, 1995,v.75,pp.89-99.DOI:https://doi.org/10.1016/01672738(94)00152-i

21. Daniels J., Wemicke R. New Aspects of an Improved PTC Model. Philips Res. Rep., 1976, v. 31, pp. 544-559.

22. Vikrant K. S. N., Edwin G. R. Charged grain boundary transitions in ionic ceramics for energy applications. Computational Materials, 2019, v. 5(1),pp. 24. DOI: https://doi.org/10.1038/s41524-019-0159-2

23. Kim M., Duscher G., Browning N. D., Sohlberg K., Pantelides S.T., Pennycook S.J. Nonstoichiometry and the electrical activity of grain boundaries in $\mathrm{SrTiO}_{3}$. Physical Review Letters, 2001, v. 86, pp. 40564059. DOI: https://doi.org/10.1103/physrevlett.86.4056

24. Oyama T., Wada N., Takagi H. Trapping of oxygen vacancy at grain boundary and its correlation with local atomic configuration and resultant excess energy in barium titanate: A systematic computational analysis. Physical Review B, 2010, v. 82, pp. 134107. DOI: https://doi.org/10.1103/physrevb.82.134107

25. Duffy D. M., Tasker P. W. Space-charge regions around dipolar grain boundaries. Journal of Applied Physics, 1984, v. 56, pp. 971-977. DOI: https://doi. org/10.1063/1.334037

26. Darinsky B. M., Efanova N. D., Prizhimov A. S. Systematics of coincidence site lattices for BCC and FCC crystals // Konsensirovannye sredy i mezhfaznye granicy [Condensed Matter and Interphases], 2018, v. 20(4), pp. 581-586. DOI: https://doi.org/10.17308/ kcmf.2018.20/632 (in Russ.)
Даринский Борис Михайлович - д. ф.-м. н., профессор, профессор кафедры материаловедения и индустрии наносистем, Воронежский государственный университет, Воронеж, Российская Федерация; e-mail: darinskii@mail.ru.ORCID iD: https://orcid.org/0000-0003-0780-9040

Ефанова Наталия Дмитриевна - студент физического факультета, Воронежский государственный университет, Воронеж, Российская Федерация; e-mail: efanowanatalia@gmail.com.

Прижимов Андрей Сергеевич - к. ф.-м. н., доцент кафедры материаловедения и индустрии наносистем, Воронежский государственный университет, Воронеж, Российская Федерация; e-mail: rnileme@mail.ru. ORCID iD: https://orcid. org/0000-0003-0052-0826
Boris M. Darinskiy - Dr. Sci. (Phys.-Math.), Full Professor, Voronezh State University, Voronezh, Russian Federation; e-mail: darinskii@mail.ru. ORCID iD: https://orcid.org/0000-0003-07809040

Natalia D. Efanova - student of Physics Faculty, Voronezh State University, Voronezh, Russian Federation; e-mail: efanowanatalia@gmail.com.

Andrey S. Prizhimov - Cand. Sci. (Phys.-Math.), Senior Researcher, Voronezh State University Voronezh, Russian Federation; e-mail: rnileme@ mail.ru. ORCID iD: https://orcid.org/0000-00030052-0826 